\title{
Magnetic microphase inhomogeneity as a thermodynamic precursor of ground-state phase separation in weakly coupled spin- $\frac{3}{2}$ chains
}

\author{
L. Shen $\odot,{ }^{1, *}$ E. Campillo $\odot,{ }^{1}$ E. Young, ${ }^{2}$ C. Bulbucan, ${ }^{1}$ R. Westerström, ${ }^{1}$ M. Laver,,${ }^{3,4}$ P. J. Baker $\odot,{ }^{5, \dagger}$ and E. Blackburn ${ }^{1, \ddagger}$ \\ ${ }^{1}$ Division of Synchrotron Radiation Research, Lund University, SE-22100 Lund, Sweden \\ ${ }^{2}$ School of Metallurgy and Materials, University of Birmingham, Birmingham B15 2TT, United Kingdom \\ ${ }^{3}$ School of Physics and Astronomy, University of Birmingham, Birmingham B15 2TT, United Kingdom \\ ${ }^{4}$ Department of Physics, University of Warwick, Coventry CV4 7AL, United Kingdom \\ ${ }^{5}$ ISIS Pulsed Neutron and Muon Source, STFC Rutherford Appleton Laboratory, \\ Harwell Oxford, Oxfordshire OX11 0QX, United Kingdom
}

(Received 28 October 2019; revised 4 June 2020; accepted 2 April 2021; published 14 April 2021)

\begin{abstract}
$\gamma-\mathrm{CoV}_{2} \mathrm{O}_{6}$ is a quasi-one-dimensional spin- $\frac{3}{2}$ magnet that possesses two distinct magnetic orders at low temperatures with propagation vectors $k_{1}=\left(\frac{1}{2}, 0,0\right)$ and $k_{2}=\left(\frac{1}{4}+\delta h, \delta k,-\frac{1}{4}+\delta l\right)$, respectively. Here we use muon spin relaxation and rotation to reveal the thermodynamics of the magnetic phase separation in this compound. In the paramagnetic (PM) region, short-range correlated spin clusters emerge at $T_{\mathrm{m}} \simeq 29 \mathrm{~K}$ at the partial expense of the PM volume. Upon further cooling, we show that these emergent clusters become spatially coherent and account for the $k_{2}$ phase below $T_{\mathrm{N} 2}=7.5 \mathrm{~K}$, while the remaining PM spins are driven into the $k_{1}$ state at $T_{\mathrm{N} 1}=6.6 \mathrm{~K}$. These results stress magnetic microphase inhomogeneity as a thermodynamic precursor for the ground-state phase separation in weakly coupled spin- $\frac{3}{2}$ chains.
\end{abstract}

DOI: 10.1103/PhysRevB.103.134420

\section{INTRODUCTION}

Quasi-one-dimensional (1D) magnets form a fertile ground to explore magnetism in low dimensions [1,2]. Typically, materials of this class develop short-range 1D spin-spin correlations, the nature of which is determined by the strength of the coupling along the chain, at elevated temperatures. The coupling between the neighboring chains, despite being significantly weaker than the intrachain coupling, will give rise to three-dimensional (3D) coherence when it becomes energetically relevant upon cooling. Recently, the exotic states of matter caused by this 1D-3D dimensional crossover have been extensively explored in both $S=\frac{1}{2}$ [3-5] and $S>\frac{1}{2}$ [6] spin systems.

The frustrated quantum many-body interactions in correlated-electron systems can lead to spatially inhomogeneous electronic or magnetic states [7,8]. In quasi-1D magnets, magnetic phase separation is often dynamic $[9,10]$ or appears in the critical region of a first-order transition [11,12] and is therefore regarded as metastable. Static phase separation in the long-range ordered magnetic ground state, on the other hand, is much rarer [13]. Moreover, thermodynamic information about the phase separation, which is essential to extract the fundamental physics in

\footnotetext{
*lingjia.shen@sljus.lu.se

†peter.baker@stfc.ac.uk

‡elizabeth.blackburn@sljus.lu.se
}

Published by the American Physical Society under the terms of the Creative Commons Attribution 4.0 International license. Further distribution of this work must maintain attribution to the author(s) and the published article's title, journal citation, and DOI. Funded by Bibsam. relevant systems [7,8], has not been understood in any quasi-1D case so far.

The triclinic cobaltate compound $\gamma-\mathrm{CoV}_{2} \mathrm{O}_{6}(\gamma \mathrm{CVO})$ has weakly coupled zigzag chains of Co running along the $b$ axis; each chain is composed of two crystallographically inequivalent $\mathrm{Co}^{2+}\left(S=\frac{3}{2}\right)$ cations, $\mathrm{Co}(1)$ and $\mathrm{Co}(2)$, in a ratio of $1: 2$ [Fig. 1(a)] [13,14]. The magnetic structure of $\gamma \mathrm{CVO}$ has been determined by neutron powder diffraction (NPD) $[13,15]$. In the ground state, this compound possesses two spatially separated magnetic orders modulated by $k_{1}=$ $\left(\frac{1}{2}, 0,0\right)$ and $k_{2}=\left(\frac{1}{4}+\delta h, \delta k,-\frac{1}{4}+\delta l\right)(\delta h=\delta k=\delta l=0)$, respectively [Fig. 1(b)] [13,16]. As the temperature increases, the $k_{2}$ phase, in which half of the Co spins are disordered, undergoes a commensurate-incommensurate transition at $T^{\star}$ $=5.6 \mathrm{~K}$, accompanied by the loss of the perfect long-range spin correlations. The $k_{1}$ phase disappears at $T_{\mathrm{N} 1}=6.6 \mathrm{~K}$, while the Néel temperature of the $k_{2}$ phase $\left(T_{\mathrm{N} 2}\right)$ could not be unambiguously determined by NPD. This is due to the prevailing magnetic diffuse scattering that emerges around the $k_{2}$ modulated Bragg positions above $T^{\star}$ and remains observable up to at least $25 \mathrm{~K}$ [13]. In the same region, another investigation using magnetometry and inelastic neutron scattering (INS) has revealed strong ferromagnetic (FM) fluctuations in this compound [15].

In a muon spin relaxation and rotation $(\mu \mathrm{SR})$ experiment, almost $100 \%$ spin-polarized positive muons are implanted inside the sample and stop rapidly at the interstitial sites. The muons decay with a mean lifetime of $\tau_{\mu}=2.2 \mu \mathrm{s}$, emitting positrons that are asymmetrically distributed in the forward and backward directions of the initial muon spin. This asymmetry can be used to determine the time evolution of the muon polarization, which is an extremely sensitive probe of the local magnetic environment $[17,18]$ and ideally suited for the study of magnetic phase separation [19-21]. 
(a)

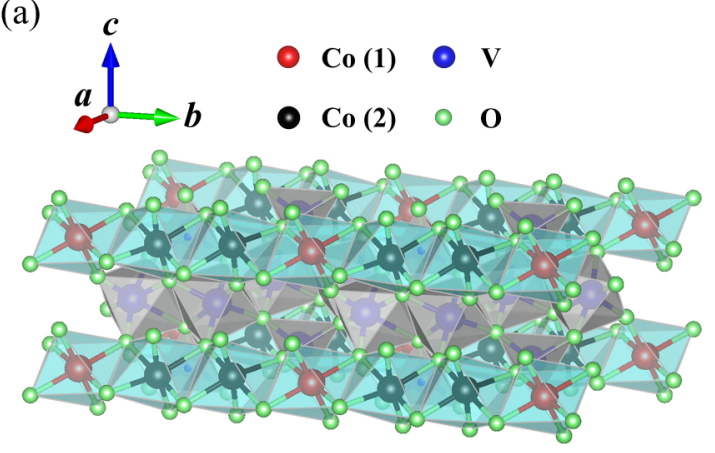

(b)

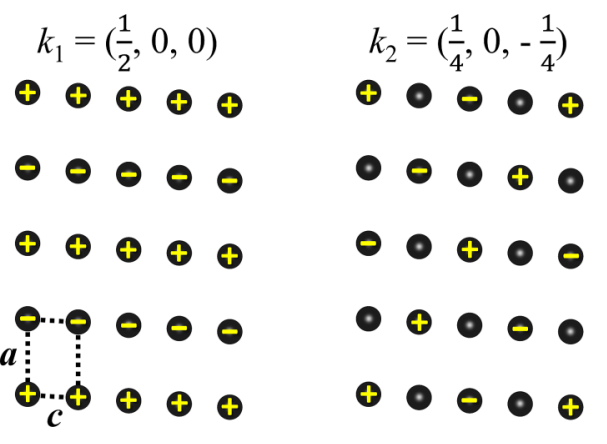

FIG. 1. Crystallographic and magnetic structures of $\gamma \mathrm{CVO}$. (a) Edge-sharing $\mathrm{CoO}_{6}$ octahedra, which are well separated by a $\mathrm{VO}_{4}-\mathrm{VO}_{6}$ polyhedral network in the $a c$ plane, propagate along the $b$ axis. (b) The ground-state spin configurations in the $a c$ plane. These planes couple ferromagnetically along the $b$ axis [13]. The \pm signs and dashed lines mark the relative spin directions and one nuclear unit cell, respectively.

In this paper, we present a detailed $\mu \mathrm{SR}$ investigation on $\gamma$ CVO. Upon cooling, the spatially homogeneous paramagnetic (PM) fluctuations break down (partially) at $T_{\mathrm{m}} \simeq$ $29 \mathrm{~K}$, as evidenced by the detection of two muon stopping environments that can be associated with the formation of local spin clusters in the PM matrix. While the zero-field (ZF) muon relaxation rate probing the residual PM volume does not diverge until the onset of the $k_{1}$ phase at $T_{\mathrm{N} 1}=6.6 \mathrm{~K}$, the one probing these emergent magnetic microphases diverges at $7.5 \mathrm{~K}\left(T_{\mathrm{N} 2}\right)$, supporting a second magnetic phase transition into the $k_{2}$ phase at this temperature. These results unveil a nontrivial thermodynamic pathway to the ground-state magnetic phase separation in weakly coupled spin- $-\frac{3}{2}$ chains.

\section{EXPERIMENTAL METHODS}

Powders of $\gamma \mathrm{CVO}$ were synthesized by the solid-state reaction method. A stoichiometric mixture of $\mathrm{V}_{2} \mathrm{O}_{5}(4 \mathrm{~N})$ and $\mathrm{CoC}_{2} \mathrm{O}_{4} \cdot 2 \mathrm{H}_{2} \mathrm{O}(4 \mathrm{~N})$ was homogeneously ground in an agate mortar, pressed into pellets, and annealed at $640{ }^{\circ} \mathrm{C}$ for six days. X-ray powder diffraction measurements were performed using a STOE STADI MP diffractometer $\left(\mathrm{Cu} \mathrm{K} \mathrm{K}_{\alpha 1}\right.$, $\lambda=1.5406 \AA$ ) to confirm the crystallographic structure of our sample at room temperature. A tiny amount of impurity phase, identified as $\mathrm{Co}_{2} \mathrm{~V}_{2} \mathrm{O}_{7}$ [22], could be resolved in our Rietveld refinement (see Appendix A); its volume fraction $(<0.5 \%)$ is well below the $\mu$ SR sensitivity threshold. The magnetic susceptibility measurements were performed in a
Quantum Design MPMS3 Superconducting Quantum Interference Device (SQUID) magnetometer. $\mu$ SR measurements were carried out on the MuSR instrument at the ISIS pulsed muon and neutron spallation source. Seven cylindrical pellets $(\sim 10 \mathrm{~mm}$ in diameter and $1 \mathrm{~mm}$ in height) of $\gamma \mathrm{CVO}$ were mounted next to each other on a silver holder and placed in a helium cryostat with a base temperature of about $1.5 \mathrm{~K}$. The standard deviation of sample temperature variation during each spectrum collection period was between $2 \mathrm{mK}$ and $3 \mathrm{mK}$.

\section{III. $\mu$ SR DATA MODELLING}

The ZF $\mu$ SR spectra collected above and below $T_{\mathrm{N} 1}$ have been fitted by

$$
A_{\mathrm{ZF}}^{T>T_{\mathrm{N} 1}}(t)=A_{\mathrm{PM}} e^{-\left(\lambda_{\mathrm{PM}} t\right)^{\beta}}+A_{\mathrm{E}} e^{-\lambda_{\mathrm{E}} t}+A_{\mathrm{C}}^{\mathrm{ZF}}+A_{\mathrm{Ag}}
$$

and

$$
\begin{aligned}
A_{\mathrm{ZF}}^{T \leqslant T_{\mathrm{N} 1}}(t)= & A_{1} e^{-\lambda_{1} t} \cos \left(2 \pi f_{1} t+\Phi_{1}\right)+A_{2} e^{-\lambda_{2} t} \\
& +A_{3} e^{-\lambda_{3} t}+A_{\mathrm{C}}^{\mathrm{ZF}}+A_{\mathrm{Ag}} .
\end{aligned}
$$

In Eq. (1), for $T>T_{\mathrm{N} 1}$, the first term describes the muons stopping in a homogeneous PM environment, where the exponent $\beta$ reflects the form of magnetic field distribution therein [23]. The second term is only resolvable for $T \leqslant$ $T_{\mathrm{m}} \simeq 29 \mathrm{~K}$; it is introduced to capture the magnetic fluctuations in the emergent spin clusters reported in Refs. [13,15].

In Eq. (2), for $T \leqslant T_{\mathrm{N} 1}$, the first term describes the coherent muon precession about the transverse quasistatic field generated by the magnetic long-range order in the $k_{1}$ phase. The second term is an approximation to the fast processes that cannot be distinguished in the current study; we will address them in Appendix B. The third term, to be discussed in Sec. IV (C), describes the magnetic fluctuations in the $k_{2}$ phase. In the modeling using Eq. (2), the phase $\Phi_{1}$ is related to the offset between the implanted muons and detector [17,20], it has been fixed to a constant value of 5.10 degrees.

The $A_{\mathrm{C}}^{\mathrm{ZF}}$ term in Eqs. (1) and (2) is only resolvable at and below about $31 \mathrm{~K}$. At $T>T_{\mathrm{N} 2}=7.5 \mathrm{~K}$, it describes the nonmagnetic muon stopping environment in $\gamma \mathrm{CVO}$. At lower temperatures, it comes from muons sensing a longitudinal local magnetic field [24,25]. The corresponding relaxation rate is so slow that this process can be effectively represented by a constant in our detection window. The $A_{\mathrm{Ag}}$ term captures the muons stopping in the silver holder. The fits on the ZF spectra were performed on the data collected between $0.14 \mu \mathrm{s}$ and $12.0 \mu \mathrm{s}$. As shown in Figs. 2(a) and 2(b) and Fig. 9 in Appendix B, Eqs. (1) and (2) well reproduce the ZF $\mu \mathrm{SR}$ spectra and resolve the muon relaxations on different timescales.

The weak transverse field (wTF) spectra were collected in a field of $20 \mathrm{G}$. In addition to the coherent muon precession caused by the external magnetic field, the spectrum is also composed of a fast relaxation and an ultra slow one. To simplify the determination of the key parameters relevant in this paper, we have omitted the data in the first $2.0 \mu \mathrm{s}$, which contain the fast component [26], and fitted the rest to the function:,

$$
A_{\mathrm{wTF}}(t)=A_{\mathrm{ex}} e^{-\lambda_{\mathrm{ex}} t} \cos \left(2 \pi f_{\mathrm{ex}} t+\Phi_{\mathrm{ex}}\right)+A_{\mathrm{C}}^{\mathrm{wTF}} .
$$



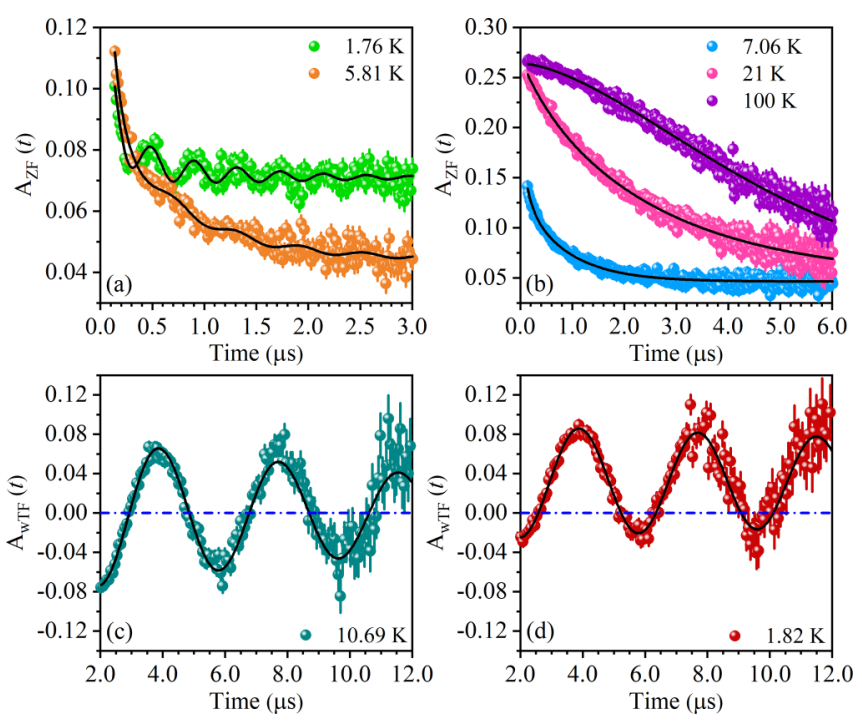

FIG. 2. Selected $\mu$ SR asymmetry spectra. (a), (b) Zero-field (ZF) measurements. (c), (d) Measurements in a weak transverse field (wTF). The wTF curves are plotted without the data collected in the initial $2.0 \mu \mathrm{s}$, which contain a fast relaxing component. The solid lines are fits using Eqs. (1)-(3). In panel (b), the shift from a Gaussian-like to Lorentzian magnetic field distribution on cooling can be seen clearly. The dash-dotted lines in (c) and (d) represent the $A_{\mathrm{C}}^{\mathrm{wTF}}$ term in Eq. (3).

In Eq. (3), the $A_{\text {ex }}$ term describes the coherent relaxation process of muons not experiencing large internal magnetic fields, i.e., the PM volume in the sample and silver sample holder [20]. The $A_{\mathrm{C}}^{\mathrm{wTF}}$ term can only be resolved below about $7.5 \mathrm{~K}$. It arises from the muons sensing a strong internal magnetic field that prevents the muon spin depolarization in the detection window [24]. As illustrated in Figs. 2(c) and 2(d), Eq. (3) reproduces the wTF $\mu$ SR spectra well.

The longitudinal field (LF) $\mu \mathrm{SR}$ spectra were collected at $1.82 \mathrm{~K}$ up to $3100 \mathrm{G}$. These fields are below the sharp metamagnetic transition at $3600 \mathrm{G}$ in this compound [15], so the information extracted should be relevant to the magnetic ground state. How the muon decay asymmetry varies with the LF depends on the field distribution within the sample and whether the fields are static [27] or dynamic [28,29]. This is particularly clear if the asymmetry projected along the initial muon spin direction is considered. To simplify the analysis and get the most essential parameter relevant in these LF measurements, we fitted the data after $1.0 \mu \mathrm{s}$ to a constant term $A_{\mathrm{C}}^{\mathrm{LF}}$. The supporting results are presented in Appendix B.

\section{RESULTS}

\section{A. Magnetic microphase separation in the paramagnetic region}

We first discuss the ZF muon relaxation between $36 \mathrm{~K}$ and $100 \mathrm{~K}$. In this region, we cannot resolve a finite $A_{\mathrm{E}}$ or $A_{\mathrm{C}}^{\mathrm{ZF}}$ value, pointing to a magnetically homogeneous state. The first term in Eq. (1) therefore accounts for the whole $\gamma \mathrm{CVO}$ sample, the volume of which is proportional to the initial asymmetry $A_{\mathrm{PM}}$ [17]. $A_{\mathrm{PM}}$ is found to be temperature independent and equal to $0.222(1)$. Moreover, the silver holder probed by the muons $\left(A_{\mathrm{Ag}}\right)$ underlies the nonrelaxing

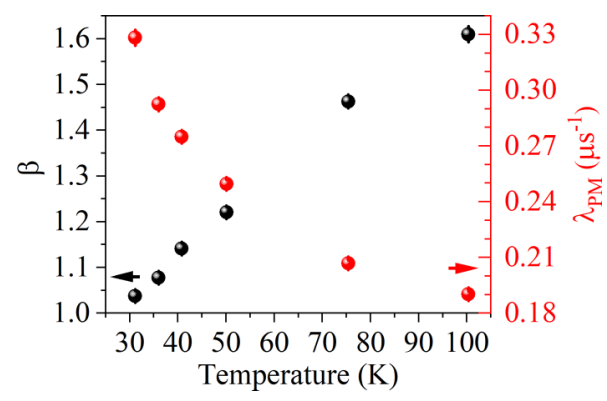

FIG. 3. Magnetic fluctuations in the paramagnetic region. Temperature dependencies ( $T>T_{\mathrm{m}} \simeq 29 \mathrm{~K}$ ) of (left axis) the exponent $\beta$ and (right axis) relaxation rate $\lambda_{\mathrm{PM}}$. These parameters are defined in Eq. (1).

component observed in the homogeneous state. $A_{\mathrm{Ag}}$ has been determined to be $0.042(1)$. In Eqs. (1) and (2), the constant terms $A_{\mathrm{Ag}}$ and $A_{\mathrm{C}}^{\mathrm{ZF}}$ are exchangeable, meaning that $A_{\mathrm{Ag}}$ can no longer be independently determined once $A_{\mathrm{C}}^{\mathrm{ZF}}$ becomes nonzero. In the numerical modelling for the $\mu \mathrm{SR}$ spectra below $36 \mathrm{~K}$, we have fixed $A_{\mathrm{Ag}}$ at 0.042 , i.e., assuming the silver sample holder contribution to be temperature independent, while its uncertainty $( \pm 0.001)$, which is primarily determined by the counting statistics, is used to define the noise level of extracting $A_{\mathrm{C}}^{\mathrm{ZF}}$ [see Figs. 4(a) and 6(a)]. We have also performed $\mathrm{ZF}$ and wTF $\mu$ SR calibration measurements on an empty silver sample holder; its relaxation effect at low temperatures is below the noise level and therefore can be ignored.

The temperature dependencies of $\beta$ and $\lambda_{\mathrm{PM}}$ are shown in Fig. 3. At about $100 \mathrm{~K}$, the highest temperature measured in
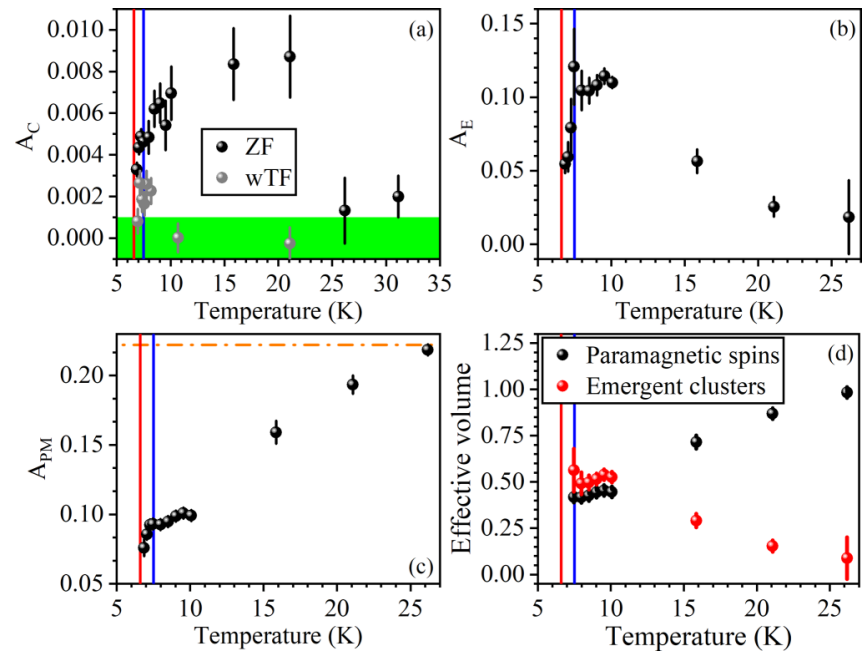

FIG. 4. Development of magnetic microphase separation. (a)(c) Temperature dependencies of the initial asymmetries described by the $A_{\mathrm{C}}^{\mathrm{ZF}}, A_{\mathrm{E}}$, and $A_{\mathrm{PM}}$ terms in Eq. (1), respectively. The $A_{\mathrm{C}}^{\mathrm{wTF}}$ term in Eq. (3) is also plotted in (a). (d) Effective volumes of the paramagnetic spins and emergent spin clusters as a function of temperature; their definitions are available in the main text. The red and blue vertical solid lines mark the positions of $T_{\mathrm{N} 1}$ and $T_{\mathrm{N} 2}$. The green area in (a) is the noise level in our measurements. The orange horizontal dash-dot line in (c) is the total sample volume. 

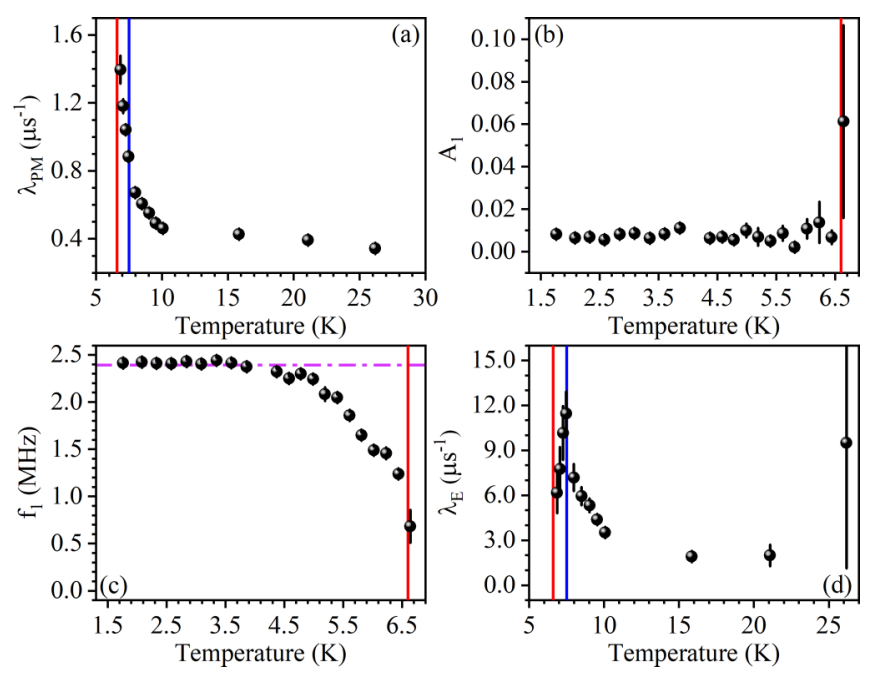

FIG. 5. Development of static magnetic ordering. Temperature dependences of the (a) paramagnetic spin relaxation rate, (b) initial asymmetry $A_{1}$, (c) coherent oscillation frequency $f_{1}$, and (d) ferromagnetic cluster relaxation rate. These parameters are described in Eq. (1). The red and blue vertical solid lines mark the positions of $T_{\mathrm{N} 1}$ and $T_{\mathrm{N} 2}$. The magenta horizontal dash-dot line in (c) is a guide to the eye.

our study, $\beta$ is 1.61(1) and shows no sign of saturation. As the temperature decreases, $\beta$ is suppressed, reaching 1.03(1) at $31 \mathrm{~K}$. At $26 \mathrm{~K}, \beta$ is further reduced to 1.0 and stops decreasing upon further cooling within the resolution. This implies that there is no distribution of relaxation times or couplings in the PM volume [17]. Therefore, we have fixed $\beta$ to 1.0 at
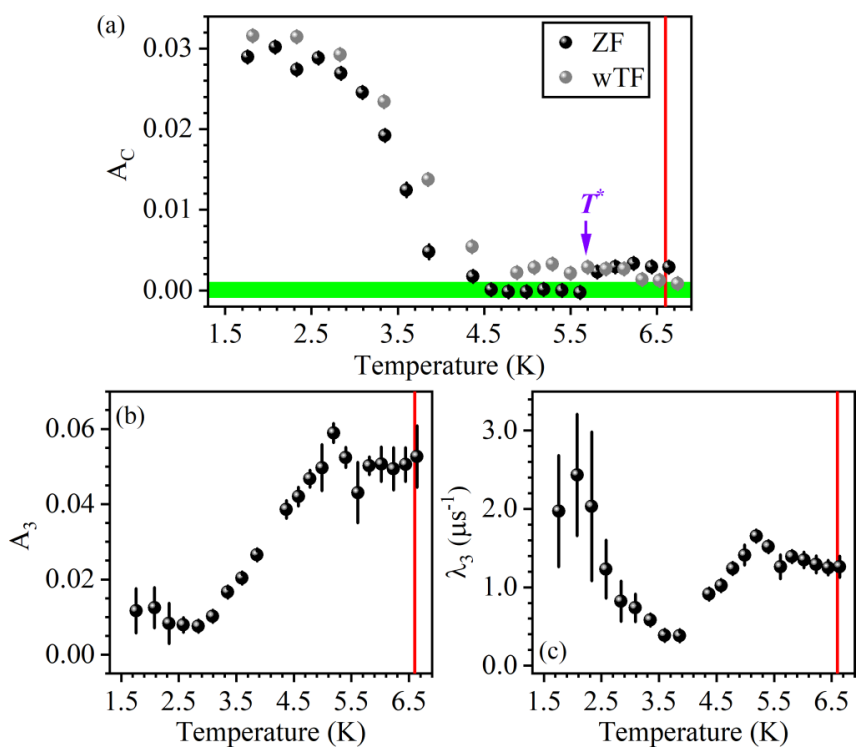

FIG. 6. Magnetic fluctuations in the magnetically ordered state. (a) Temperature dependencies of the $A_{\mathrm{C}}^{\mathrm{ZF}}$ and $A_{\mathrm{C}}^{\mathrm{wTF}}$ terms in Eqs. (2) and (3), respectively. The green area is the noise level. $T^{\star}$ is the temperature where the $k_{2}$ phase undergoes an incommensuratecommensurate lock-in transition [13]. (b), (c) The $A_{3}$ and $\lambda_{3}$ terms in Eq. (2) as a function of temperature. The red lines mark the position of $T_{\mathrm{N} 1}$. lower temperatures. The evolution of $\beta$ can be explained by a change in the nature of the dynamics probed by the muons. At high temperatures, $\beta=2.0$ comes from the random and isotropic fields that dominate the spin relaxation. These fields are probably mostly nuclear in origin. On cooling, the magnetic correlations become stronger and therefore electronic relaxation dominates the signal. This process reduces $\beta$ to 1.0. These are likely to be the Ising-like correlations along the effectively isolated spin chains in $\gamma \mathrm{CVO}$ [13]. A similar effect has been observed in the Ising-like system $\mathrm{BaCo}_{2} \mathrm{~V}_{2} \mathrm{O}_{8}$ [23]. This scenario fits with the magnetic susceptibility of our sample, which reveals deviation from the Curie-Weiss (CW) description of a completely disordered spin system well above $T_{\mathrm{m}}$ [ $\sim 250 \mathrm{~K}$, see Fig. 12(b) in Appendix C]. $\lambda_{\mathrm{PM}}$ gradually increases upon cooling; it agrees with the typical behavior of muons stopping in a PM environment and will be revisited in more detail later.

A finite $A_{\mathrm{C}}^{\mathrm{ZF}}$ value of $0.002(1)$ in Eq. (1) can be obtained at $31 \mathrm{~K}$, while the $A_{\mathrm{E}}$ term still remains below the detection level; we have fixed the latter at zero in the numerical fitting. At $26 \mathrm{~K}$ and below, both $A_{\mathrm{C}}^{\mathrm{ZF}}$ and $A_{\mathrm{E}}$ become resolvable [Figs. 4(a) and 4(b)]. Concomitantly, the PM asymmetry in the sample begins to decrease [Fig. 4(c)] due to the growth of the emergent spin clusters $[13,15]$ described by these terms. Because there is no magnetic long-range order at these temperatures [13], our observations strongly support the onset of magnetic microphase separation in this material at $T_{\mathrm{m}}=$ $\frac{31 \mathrm{~K}+26 \mathrm{~K}}{2} \simeq 29 \mathrm{~K}$.

The effective volumes of the PM spins and emergent spin clusters below $T_{\mathrm{m}}$ can be calculated by $\frac{A_{\mathrm{PM}}}{A_{\mathrm{tot}}}$ and $\frac{A_{\mathrm{C}}^{\mathrm{ZF}}+A_{\mathrm{E}}}{A_{\mathrm{tot}}}$, respectively, where $A_{\text {tot }}=0.222(1)$ is the total sample asymmetry obtained from the measurements above $T_{\mathrm{m}}$. Their temperature dependencies are shown in Fig. 4(d), which reveal that the development of the emergent spin clusters is at the partial expense of the PM spins.

The constant $A_{\mathrm{C}}^{\mathrm{ZF}}$ term can arise either from a nonmagnetic muon stopping environment or a large internal longitudinal magnetic field that prevents muon depolarization [24]. To understand its nature, we analyze the wTF spectra using Eq. (3). As shown in Fig. 4(a), $A_{\mathrm{C}}^{\mathrm{wTF}}$ only becomes marginally resolvable close to the critical region near $7.5 \mathrm{~K}$. Accordingly, the distinct temperature dependencies of $A_{\mathrm{C}}^{\mathrm{ZF}}$ and $A_{\mathrm{C}}^{\mathrm{wTF}}$ between $T_{\mathrm{N} 2}$ and $T_{\mathrm{m}}$ means that $A_{\mathrm{C}}^{\mathrm{ZF}}$ has a nonmagnetic contribution in this region, which is absorbed by the precession term $A_{\mathrm{ex}}$ in Eq. (3) in the presence of a wTF. The $A_{\mathrm{C}}^{\mathrm{ZF}}$ and $A_{\mathrm{C}}^{\mathrm{wTF}}$ terms below $7.5 \mathrm{~K}$ will be discussed in Sec. IV C.

\section{B. Thermodynamic path to the ground-state magnetic phase separation}

The PM fluctuation rate $\left[\lambda_{\mathrm{PM}}\right.$ in Eq. (1)] diverges while approaching $T_{\mathrm{N} 1}=6.6 \mathrm{~K}$ [Fig. 5(a)], below which a coherent oscillation described by the $A_{1}$ term in Eq. (2) is observed [Figs. 5(b) and 5(c)], signifying a magnetic phase transition. The temperature dependence of $f_{1}$ term fits the character of the $k_{1}$ phase, as revealed by NPD [13]. Moreover, combining this with the fact that only the muon relaxation rate describing the PM volume diverges at $T_{\mathrm{N} 1}$, we conclude that the spins in the PM volume condense into the $k_{1}$ state at $T_{\mathrm{N} 1}=6.6 \mathrm{~K}$. 
The temperature dependence of the muon relaxation rate describing the emergent spin clusters $\left(\lambda_{\mathrm{E}}\right)$ is shown in Fig. 5(d). Unlike the PM spins, the magnetic fluctuations generated by these short-range correlated clusters diverge at a higher temperature: $T_{\mathrm{N} 2}=7.5 \mathrm{~K}$. Concomitantly, $A_{\mathrm{E}}$ and $A_{\mathrm{C}}^{\mathrm{ZF}}$ are suppressed [Figs. 4(a) and 4(b)]. These features suggest the existence of a second magnetic phase transition at $T_{\mathrm{N} 2}$. The parameters describing these clusters evolve smoothly near $T_{\mathrm{N} 1}$. This implies a loose coupling between them and the spins in the $k_{1}$ domain. Based on these observations, we assign $T_{\mathrm{N} 2}$ to the onset of the $k_{2}$ phase. Additional support for this assignment comes from the magnetic susceptibility versus temperature measurements, which show a broad peak centered at $T_{\mathrm{N} 2}$ in our sample[Fig. 12(a) in Appendix C]. All these can be explained by the prevailing local FM clusters in the $k_{2}$ volume between $T_{\mathrm{N} 2}$ and $T_{\mathrm{m}}[13,15]$, the net moment of which only gets canceled out below its Néel temperature.

The data presented above allow us to establish a clear thermodynamic path to the ground-state magnetic phase separation in $\gamma \mathrm{CVO}$ upon cooling: Magnetic microphase separation occurs at $T_{\mathrm{m}}$. The emergent spin clusters described by the $A_{\mathrm{E}}$ and $A_{\mathrm{C}}^{\mathrm{ZF}}$ terms in Eq. (1) account for the $k_{2}$ phase at $T_{\mathrm{N} 2}$, while the remaining PM spins described by the $A_{\mathrm{PM}}$ term form the $k_{1}$ phase at $T_{\mathrm{N} 1}$.

\section{Magnetic fluctuations below $\boldsymbol{T}_{\mathrm{N} 2}$}

In Eq. (2), the $A_{1}$ term has an initial asymmetry of $0.008(1)$ in the ground state [Fig. 5(b)]; it is due to the muons sensing a transverse quasistatic magnetic field in the $k_{1}$ phase. In a randomly oriented sample, the initial asymmetry of an oscillating term amounts to $\frac{2}{3}$ of the total volume probed by these muons [24,25]. Accordingly, the effective volume responsible is $5.4(7) \%$. This value is far below the volume fraction of the $k_{1}$ phase [65(1)\%] determined by neutron diffraction [13]. As discussed in Appendix B, this discrepancy is caused by the existence of fast processes below $T_{\mathrm{N} 2}$ that are beyond our detection limit. As a result, an accurate determination of the relevant phase volumes in the ground state is not possible with the current data; further studies are required to shed light on these missing dynamics.

$A_{\mathrm{C}}^{\mathrm{ZF}}$ has a nonvanishing contribution from the sample below $T_{\mathrm{m}}$ [Fig. 4(a)]. It starts to decrease below $T_{\mathrm{N} 2}$, while $A_{\mathrm{C}}^{\mathrm{wTF}}$ becomes finite. $A_{\mathrm{C}}^{\mathrm{ZF}}$ and $A_{\mathrm{C}}^{\mathrm{wTF}}$ are almost identical between $T^{\star}=5.6 \mathrm{~K}$ and $T_{\mathrm{N} 2}$ [Figs. 4(a) and 6(a)]; this provides the evidence that they both probe a large internal longitudinal magnetic field in the $k_{2}$ domain [27]. $A_{\mathrm{C}}^{\mathrm{ZF}}$ reduces to zero at $T^{\star}$. Since this term describes the magnetic fluctuations in the $k_{2}$ phase, the suppression could be associated with the incommensurate-commensurate lock-in transition in the $k_{2}$ phase at the same temperature [13]. However, the values of $A_{\mathrm{C}}^{\mathrm{ZF}}$ above $T^{\star}$ are marginal and close to the noise level. Moreover, $A_{\mathrm{C}}^{\mathrm{wTF}}$ does not respond to $T^{\star}$. In our analysis, we have implemented the assumption that the other constant term $A_{\mathrm{Ag}}$ in Eqs. (1) and (2), which is correlated with the $A_{\mathrm{C}}^{\mathrm{ZF}}$ determination, is temperature independent below $36 \mathrm{~K}$. A tiny change in the silver sample holder position with respect to the muon beam can also lead to an artificial variation in $A_{\mathrm{C}}^{\mathrm{ZF}}$. Calibration measurements in the same cryostat on an empty silver sample holder do not show such an effect. However, since we are close to the noise level, we cannot draw a firm conclusion on the small change in $A_{\mathrm{C}}^{\mathrm{ZF}}$.

We now discuss the muon relaxation process described by the $A_{3}$ term in Eq. (2) [Figs. 6(b) and 6(c)]. First, it is not related to the $k_{1}$ phase, because it evolves smoothly in the critical region near $T_{\mathrm{N} 1}$. Moreover, its initial asymmetry is too large to account for the domain walls between the two phases. Most of all, its suppression below about $4.6 \mathrm{~K}$, at which the two magnetic phase transitions in this compound complete (see Fig. 5(c) and Ref. [13]), strongly correlates with the reentrant growth of the $A_{\mathrm{C}}^{\mathrm{ZF}}$ term [Fig. 6(a)]. We therefore assign it to the magnetic fluctuations in the $k_{2}$ phase, which are probed by the muons sensing an internal magnetic field parallel to their spin directions. At $2.8 \mathrm{~K}$ and below, the fluctuations in the $k_{2}$ phase are stabilized.

Below about $4.6 \mathrm{~K}$, the temperature dependence of $A_{\mathrm{C}}^{\mathrm{wTF}}$ resembles that of $A_{\mathrm{C}}^{\mathrm{ZF}}$ [Fig. 6(a)]. This firmly confirms that the reentrant behavior of the $A_{\mathrm{C}}^{\mathrm{ZF}}$ term is caused by the slowing down of the muon depolarization in a large internal magnetic field [24], which is parallel to the muon spin and external field directions in the ZF and wTF configurations, respectively. This also naturally connects the changes in $A_{\mathrm{C}}^{\mathrm{ZF}}$ and $A_{3}$ to the same origin at low temperatures. The residual $A_{3}$ term below $2.8 \mathrm{~K}$ [Fig. 6(b)], on the other hand, could come from the weak short-range spin correlations that persist down to at least $1.5 \mathrm{~K}$, as revealed by NPD [13]. Accordingly, the fast muon relaxation above $4.6 \mathrm{~K}$ captured by the $A_{3}$ term is related to the strong spin fluctuations in the transition region or the incommensurate spin arrangement above $T^{\star}$. These results highlight the metastable nature of the $k_{2}$ phase in this compound at intermediate temperatures, which only gets fully stabilized below $2.8 \mathrm{~K}$.

\section{DISCUSSION AND SUMMARY}

With all the results presented above, we discuss the microscopic picture of the magnetic phase separation in $\gamma \mathrm{CVO}$. Although no magnetic order is formed above $T_{\mathrm{N} 2}$, this system does not fit the CW description between $100 \mathrm{~K}$ and $T_{\mathrm{m}}$ [15]. Because of the positive Weiss temperature in our sample $(\sim$ 14.5 K, see Appendix C) [15], which comes predominantly from the intrachain coupling, we believe that local spin-spin correlations develop along the chains in this region. From our $\mu \mathrm{SR}$ data, $\gamma \mathrm{CVO}$ is magnetically homogeneous above $T_{\mathrm{m}}$ (Fig. 3), meaning that the 1D FM clusters should populate the entire sample volume. The magnetic microphase separation sets in when $\gamma \mathrm{CVO}$ is cooled below $T_{\mathrm{m}}$. If we assume that the 1D character persists on cooling, it may be that the effectively isolated spin chains become magnetically inhomogeneous, with no correlations between them, or in the ac plane. Another possibility is that a local 1D-3D crossover occurs at $T_{\mathrm{m}}$. In this scenario, the magnetic inhomogeneities described by the $A_{\mathrm{E}}$ term in Eq. (1) are exclusively introduced by the energetically relevant interchain coupling(s). Although $\mu \mathrm{SR}$ is a local probe and therefore cannot directly distinguish these scenarios alone, the higher Néel temperature for the spins in these emergent clusters $\left(T_{\mathrm{N} 2}\right)$ seems to favor the second scenario from an energy point of view. This interchain scenario can also explain the puzzle of dominant magnetic diffuse scattering around the $k_{2}$ modulated positions 


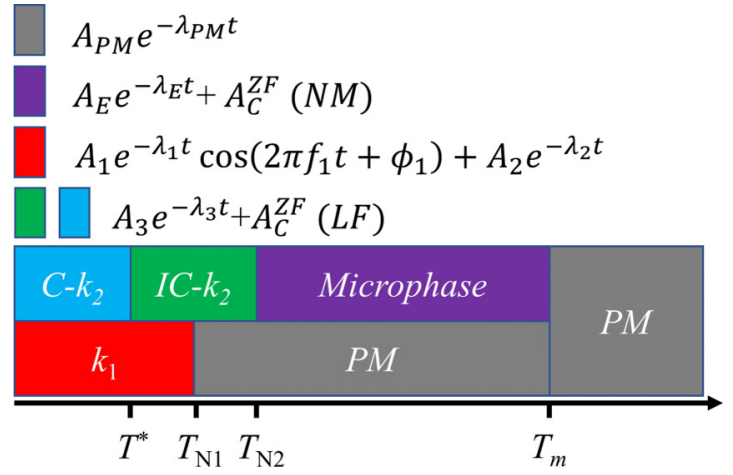

FIG. 7. The magnetic phase diagram of $\gamma \mathrm{CVO}$ as a function of temperature. C, IC, PM, NM, and LF correspond to commensurate, incommensurate, paramagnetic, nonmagnetic, and longitudinal field, respectively. The term(s) in Eqs. (1) or (2) describing the corresponding phase is (are) also displayed.

below $T_{\mathrm{m}}$ though it is a minority phase in the ground state on the neutron diffraction timescale [13]. While the spins in the emergent clusters $\left(k_{2}\right.$ domain) develop some coherence at $T_{\mathrm{N} 2}$, the true magnetic long-range order, together with its modulation vector, is not stabilized until $T^{\star}$. Although the $k_{2}$ magnetic phase transition is completed around $4.6 \mathrm{~K}$, strong magnetic fluctuations, described by the $A_{3}$ and $A_{\mathrm{C}}^{\mathrm{ZF}}$ terms in Eq. (2), can be observed down to $2.8 \mathrm{~K}$. This observation could be connected to the strong low-energy excitations above $2.0 \mathrm{~K}$ observed by INS [15]. As for the $k_{1}$ phase, it results from the nonvanishing PM volume in the magnetic microphase separation state. Since no interchain spin correlation exists in these domains, this state is energetically less favored, corresponding to a lower transition temperature $T_{\mathrm{N} 1}$. Below $T_{\mathrm{N} 1}$, the spins in this phase behave like those in a conventional antiferromagnet [17].

In summary, we have used $\mu \mathrm{SR}$, which is an extremely sensitive probe of the local magnetic environment [17], to follow the development and evolution of the spatially segregated magnetic phases in $\gamma \mathrm{CVO}$ as a function of temperature. The obtained magnetic phase diagram, along with the $\mu \mathrm{SR}$ term(s) describing the corresponding phase [Eqs. (1) and (2)],
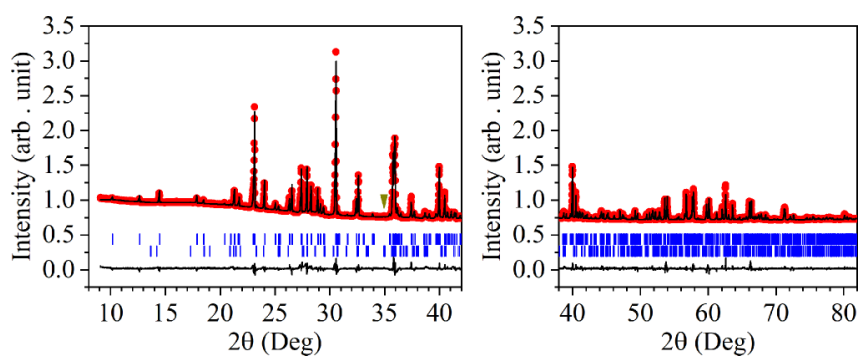

FIG. 8. The XRD patterns of $\gamma \mathrm{CVO}$ at room temperature. The red solid dots are experimental observations. The black lines are the calculated pattern (upper) and the difference between the experimental and calculated data (bottom). The blue vertical bars are the Bragg positions of $\gamma \mathrm{CVO}$ (upper) and $\mathrm{Co}_{2} \mathrm{~V}_{2} \mathrm{O}_{7}$ (bottom). The dark yellow arrow marks one weak Bragg reflection from the impurity $\mathrm{Co}_{2} \mathrm{~V}_{2} \mathrm{O}_{7}$ phase. is displayed in Fig. 7. The key finding is the magnetic microphase inhomogeneities that emerge in the PM state at $T_{\mathrm{m}}$, which we then demonstrate to be a thermodynamic precursor for the ground-state phase separation in this material. In the family of quasi-1D magnets, the static and dynamic magnetic properties in the homogeneous state have been intensively studied $[3,5,6,30]$. The physics of magnetic phase separation, however, are not well understood in materials of this class and have mostly been focused on materials with a higher effective dimension, e.g., the 2D Kagomé or triangular lattice $[9,10,31]$ and 3D perovskite lattice [7]. Regardless of the dimension, one key component shared by all these materials is the strong electron correlation effect, which leads to a situation dominated by frustrated quantum many-body interactions, in which the system's total free energy cannot be minimized by optimizing the interaction energy between every pair of spins or electrons. Our work has unveiled the thermodynamic pathway to the phase-separated magnetic ground state in a quasi-1D magnet and therefore provides a concrete foundation for future theoretical and experimental studies.

The $\mu$ SR data collected at ISIS are available at Ref. [32].

\section{ACKNOWLEDGMENTS}

We thank L. Folkers for kind assistance in the sample preparation stage. We thank Thomas Greber for giving us access to the SQUID. We also gratefully acknowledge the Science and Technology Facilities Council (STFC) for access to muon beamtime at ISIS.

\section{APPENDIX A: ROOM-TEMPERATURE X-RAY DIFFRACTION}

High-resolution x-ray diffraction measurements were performed at room temperature. The obtained pattern was refined using the Rietveld method in the FullPrOF package [33]. As shown in Fig. 8, the majority of the Bragg peaks belong

TABLE I. The refined atomic positions of $\gamma$ CVO. The lattice parameters are $a=7.1799$ (1) $\AA, b=8.8992(1) \AA, c=4.81306(7)$ $\AA, \alpha=90.2744(6)^{\circ}, \beta=93.6732(5)^{\circ}$, and $\gamma=102.1940(6)^{\circ}$, respectively.

\begin{tabular}{lclc}
\hline \hline Atom & $x$ & \multicolumn{1}{c}{$y$} & $z$ \\
\hline Co (1) & 0 & 0.5 & 0 \\
Co (2) & $0.0213(5)$ & $0.1715(4)$ & $0.0190(7)$ \\
O (1) & $0.185(1)$ & $0.503(1)$ & $0.346(2)$ \\
O (2) & $0.838(1)$ & $0.627(1)$ & $0.143(2)$ \\
O (3) & $0.177(1)$ & $0.711(1)$ & $0.865(2)$ \\
O (4) & $0.158(1)$ & $0.031(1)$ & $0.822(2)$ \\
O (5) & $0.166(1)$ & $0.880(1)$ & $0.335(2)$ \\
O (6) & $0.791(1)$ & $0.791(1)$ & $0.651(2)$ \\
O (7) & $0.479(1)$ & $0.942(1)$ & $0.684(2)$ \\
O (8) & $0.477(1)$ & $0.571(1)$ & $0.700(2)$ \\
O (9) & $0.524(1)$ & $0.753(1)$ & $0.190(2)$ \\
V (1) & $0.7178(5)$ & $0.9690(5)$ & $0.4614(8)$ \\
V (2) & $0.7156(5)$ & $0.6114(5)$ & $0.4551(8)$ \\
V (3) & $0.5936(6)$ & $0.2592(6)$ & $0.1208(7)$ \\
\hline \hline
\end{tabular}




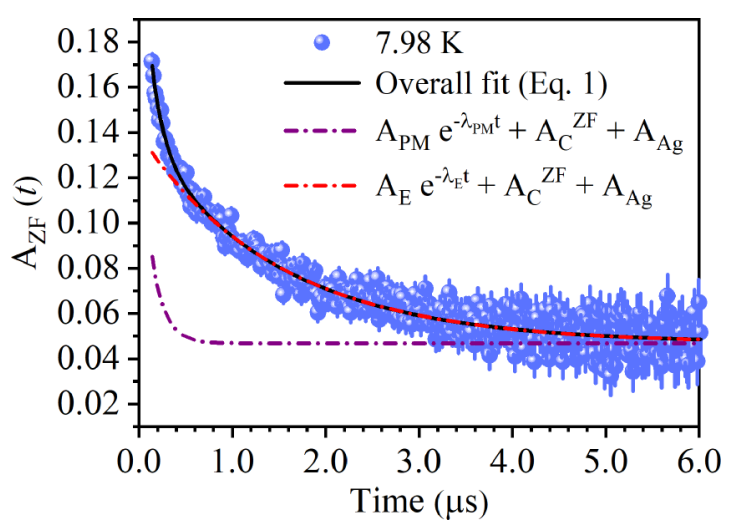

FIG. 9. Zero field (ZF) $\mu$ SR asymmetry spectrum collected at $7.98 \mathrm{~K}$. The black solid line is the overall fit using Eq. (1). The dashdotted lines represent the individual relaxations in this model offset by the constant $A_{\mathrm{C}}^{\mathrm{ZF}}+A_{\mathrm{Ag}}$.

to $\gamma \mathrm{CVO}$ with a volume fraction of $99.55(84) \%$; the refined lattice parameters and atomic positions are listed in Table I. These values are in broad agreement with the ones reported in Ref. [13]. In addition to $\gamma \mathrm{CVO}$, a very weak impurity phase with a volume fraction of $0.45(18) \%$, identified as $\mathrm{Co}_{2} \mathrm{~V}_{2} \mathrm{O}_{7}$ [22], could also be resolved (Fig. 8).

\section{APPENDIX B: DETAILED $\mu$ SR SPECTRUM ANALYSIS}

The ZF $\mu$ SR spectrum at $7.98 \mathrm{~K}$ is shown in Fig. 9, along with the individual muon relaxation processes described by Eq. (1). The good agreement between experimental observations and fit supports our contention that the different components in Eq. (1) are required to comprehensively capture the magnetic fluctuations on distinct timescales within the detection window.

The total sample asymmetry extracted in our measurements $\left(A_{\text {tot }}\right)$, i.e., $A_{\mathrm{PM}}+A_{\mathrm{E}}+A_{\mathrm{C}}^{\mathrm{ZF}}$ for $T>T_{\mathrm{N} 1}$ [Eq. (1)] and $A_{1}+A_{2}+A_{3}+A_{\mathrm{C}}^{\mathrm{ZF}}$ for $T \leqslant T_{\mathrm{N} 1}$ [Eq. (2)], is shown in Fig. 10(a). Comparing with the high-temperature values which amount to the whole sample $(\sim 0.222), A_{\text {tot }}$ gets suppressed when entering the magnetically ordered region $(T<$
$T_{\mathrm{N} 2}$ ). This suggests the development of fast dynamics that escaped our detection window.

To further elucidate the missing component, we have performed $\mu \mathrm{SR}$ measurements in a longitudinal magnetic field up to $3100 \mathrm{G}$ at $1.82 \mathrm{~K}$. These fields are below the sharp metamagnetic transition in this compound at $3600 \mathrm{G}$ [15]. The information extracted should therefore be primarily relevant to the ZF magnetic ground state. The muon spin relaxation and rotation processes described by the $A_{\mathrm{i}}(i=1,2,3)$ term in Eq. (2) cannot be well resolved within our counting statistics in the LF configuration [Fig. 10(b)]. Consequently, we have omitted the first $1.0 \mu \mathrm{s}$ in our analysis and fitted the rest by two constant terms $A_{\mathrm{C}}^{\mathrm{LF}}+A_{\mathrm{Ag}}$, where $A_{\mathrm{Ag}}=0.042(1)$ (main text). The LF dependence of $A_{\mathrm{C}}^{\mathrm{LF}}$ is shown in Fig. 10(c). Based on the decoupling scenario [27-29], at least two magnetic fields $\left(B_{\mathrm{c} 1}\right.$ and $\left.B_{\mathrm{c} 2}\right)$ are required to reproduce this curve. Assuming that both fields have a broad distribution, we modeled the data by

$$
A_{\mathrm{C}}^{\mathrm{LF}}=A_{\mathrm{C}}^{0}+A_{\mathrm{C}}^{1} \frac{b_{1}^{2}}{b_{1}^{2}+1}+A_{\mathrm{C}}^{2} \frac{b_{2}^{2}}{b_{2}^{2}+1},
$$

where $b_{i}=B / B_{\mathrm{ci}}(i=1,2), A_{\mathrm{C}}^{0}$ is the constant asymmetry in $\mathrm{ZF}$, and $A_{\mathrm{C}}^{\mathrm{i}}$ is the asymmetry associated with $B_{\mathrm{ci}}(i=$ $1,2)[28,29]$. Due to the limited data range, the fit diverges if we allow all parameters in Eq. (B1) to vary. Alternatively, we evaluate these parameters while keeping $\sum A_{C}^{\mathrm{i}}$ at a fixed value between 0.060 and 0.222 . The upper bound 0.222 is the maximum asymmetry allowed for the sample (main text); the lower bound 0.060 is used to justify that a sufficiently large $\sum A_{C}^{\mathrm{i}}$ is required to produce a satisfactory fit. At $\sum A_{\mathrm{C}}^{\mathrm{i}} \geqslant 0.100$, the quality of the fit, $A_{\mathrm{C}}^{1}$ and $B_{\mathrm{c} 1}$, are not sensitive to the choice of $\sum A_{\mathrm{C}}^{\mathrm{i}}$ [Fig. 10(c), Table II]. $B_{\mathrm{c} 1}$ corresponds to a characteristic frequency of $2.9(4) \mathrm{MHz}$, which is close to $f_{1}=2.41(2) \mathrm{MHz}$ measured in our experiment [Fig. 5(c)]. Although the large $B_{\mathrm{c} 2}$ cannot be accurately determined, its non-negligible contribution to the LF spectra is evident.

The decoupling effect demonstrated in Fig. 10(c) is unlikely of muonium origin, which has a similar form [34]. Vacuum-state muonium would have a decoupling field $B_{0}$ $=1585 \mathrm{G}$. Certain chemical states or delocalisation of
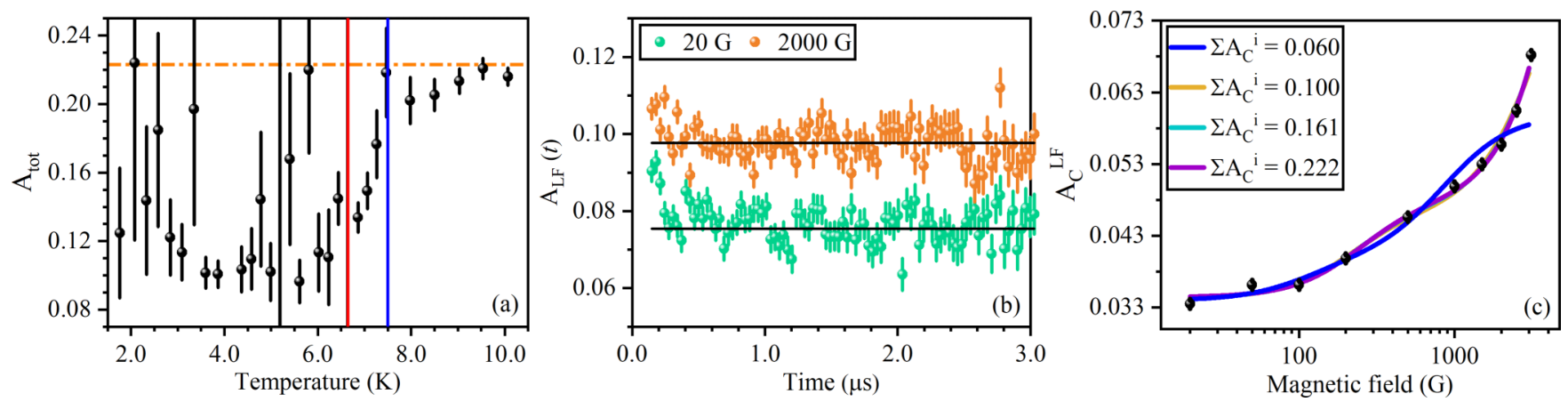

FIG. 10. (a) Temperature dependence of the total asymmetry measured $\left(A_{\mathrm{tot}}\right)$. The purple line is the total sample asymmetry determined from the high-temperature measurements. The blue and red lines mark the positions of $T_{\mathrm{N} 2}$ and $T_{\mathrm{N} 1}$, respectively. Due to the appearance of multiple fast dynamics that cannot be accurately determined within our detection window $\left[A_{2}\right.$ term in Eq. (2), also see Fig. 11(b)], $A_{\text {tot }}$ becomes less well defined below $T_{\mathrm{N} 1}=6.6 \mathrm{~K}$. (b) $\mathrm{LF} \mu \mathrm{SR}$ spectra at $1.82 \mathrm{~K}$. The solid lines are fits to two constant terms $A_{\mathrm{C}}^{\mathrm{LF}}+A_{\mathrm{Ag}}$. (c) Field dependence of $A_{\mathrm{C}}^{\mathrm{LF}}$. The solid lines are fits to Eq. (B1). 
TABLE II. Fitting parameters of the LF $\mu$ SR spectra at $1.82 \mathrm{~K}$ using Eq. (B1).

\begin{tabular}{ccccc}
\hline \hline$\sum A_{\mathrm{C}}^{\mathrm{i}}$ & $A_{\mathrm{C}}^{0}$ & $A_{\mathrm{C}}^{1}$ & $B_{\mathrm{c} 1}(\mathrm{G})$ & $B_{\mathrm{c} 2}(\mathrm{G})$ \\
\hline 0.060 & $0.034(1)$ & $0.006(9)$ & $117(280)$ & $857(457)$ \\
0.100 & $0.034(1)$ & $0.012(2)$ & $218(55)$ & $4008(240)$ \\
0.161 & $0.034(1)$ & $0.013(2)$ & $238(42)$ & $6722(266)$ \\
0.222 & $0.034(1)$ & $0.013(1)$ & $245(39)$ & $8652(310)$ \\
\hline \hline
\end{tabular}

muonium can lead to lower decoupling fields, but not larger ones. These values are well within our measurement range so we would directly observe a different signal if the asymmetry loss were due to muonium. In theory, the $B_{\mathrm{c} 1}$ in Table II could be a muonium state. However, the observation of a $\mathrm{ZF}$ coherent precession close to this value [Fig. 5(c)] makes it exceptionally difficult to reconcile with the presence of muonium. $B_{\mathrm{c} 2}$ is several times higher than $B_{0}$ and therefore cannot be a muonium state. In fact, its value is comparable with that observed in $\mathrm{Co}_{3} \mathrm{O}_{4}$ [35]. Its presence supports our proposal that there exists large quasistatic magnetic fields or significant dynamics that exceed the experimental resolution.

We also performed additional fitting to the $\mu \mathrm{SR}$ spectra below $2.8 \mathrm{~K}$. Here, we have assumed that the parameters $A_{1}, A_{2}, A_{3}$, and $A_{\mathrm{C}}^{\mathrm{ZF}}$ in Eq. (2) are temperature independent because the magnetic fluctuations probed by muons are fully stabilized in this region (see the results in the main text). For comparison, $\lambda_{1}, \lambda_{2}$, and $A_{2}$ obtained with and without the above additional constraint are plotted in Fig. 11. It is evident that this approximation significantly reduces the errors of these parameters. In the following, we use this approach to discuss the $A_{2}$ term in the ground state $(T \leqslant 2.8 \mathrm{~K})$, which is $0.102(4)$ and must contain at least two fast dynamics that cannot be distinguished within our resolution. If we assume that it exclusively comes from the incoherent muon precession about the transverse magnetic field in the $k_{2}$ phase, $A_{2}$ would amount to twice the initial asymmetries capturing the longitudinal magnetic field therein [20], i.e., $\left(A_{\mathrm{C}}^{\mathrm{ZF}}+A_{3}\right) \times 2$ $=0.058(4)$. This value alone is not large enough to explain our observation. On the other hand, the $A_{2}$ term could also describe the longitudinal magnetic field in the $k_{1}$ phase. Our
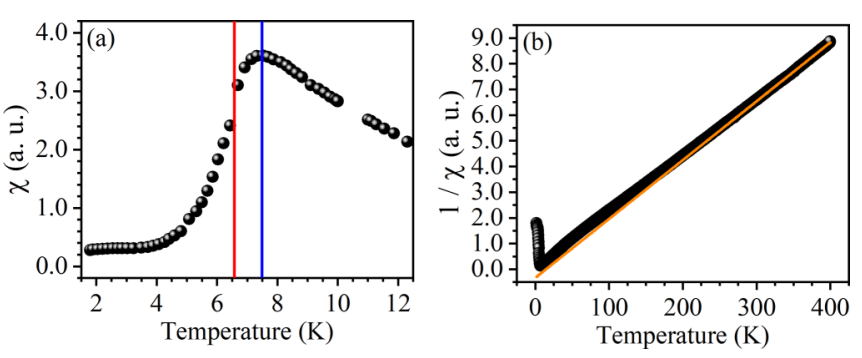

FIG. 12. Magnetic susceptibility $(\chi)$ of $\gamma$ CVO. (a) $\chi$ versus temperature measured at $0.01 \mathrm{~T}$ and below $12.5 \mathrm{~K}$. The red and blue solid lines mark the positions of $T_{\mathrm{N} 1}=6.6 \mathrm{~K}$ and $T_{\mathrm{N} 2}=7.5 \mathrm{~K}$, respectively. (b) Inverse $\chi$ versus temperature curve up to $400 \mathrm{~K}$. The orange solid line is the Curie-Weiss fit based on the data points between $350 \mathrm{~K}$ and $400 \mathrm{~K}$. These data were collected on warming.

measurements are able to reveal a quasistatic transverse magnetic field described by the $A_{1}$ term in Eq. (2) [Figs. 5(b), 5(c), and 11(a)]. The corresponding longitudinal component only generates a small initial asymmetry of $A_{1} \times 0.5=0.0040(5)$, which again fails to explain the observed $A_{2}$ value. Since the LF $\mu$ SR measurements strongly support the existence of fast dynamics that are beyond our detection limit ( $B_{\mathrm{c} 2}$ in Table II), it is likely that they contribute to the missing asymmetries in $A_{2}$.

\section{APPENDIX C: MAGNETIZATION VERSUS TEMPERATURE}

The magnetic susceptibility $(\chi)$ of $\gamma \mathrm{CVO}$ has been measured as a function of temperature (on warming) at $0.01 \mathrm{~T}$. As shown in Fig. 12(a), the broad peak in $\chi$ is centered at $7.5 \mathrm{~K}$; this value matches $T_{\mathrm{N} 2}$ extracted from the $\mu \mathrm{SR}$ measurements (see the discussion in the main text). We have also plotted out the $1 / \chi$ versus temperature curve in Fig. 12(b). A CW fit has been performed on the data points between $350 \mathrm{~K}$ and $400 \mathrm{~K}$, which produces a positive Weiss temperature of about $14.5 \mathrm{~K}$. This agrees with the dominant FM intrachain spin exchange interactions in $\gamma \mathrm{CVO}$ [15]. Moreover, a deviation from the CW behavior is observed above $200 \mathrm{~K}$.
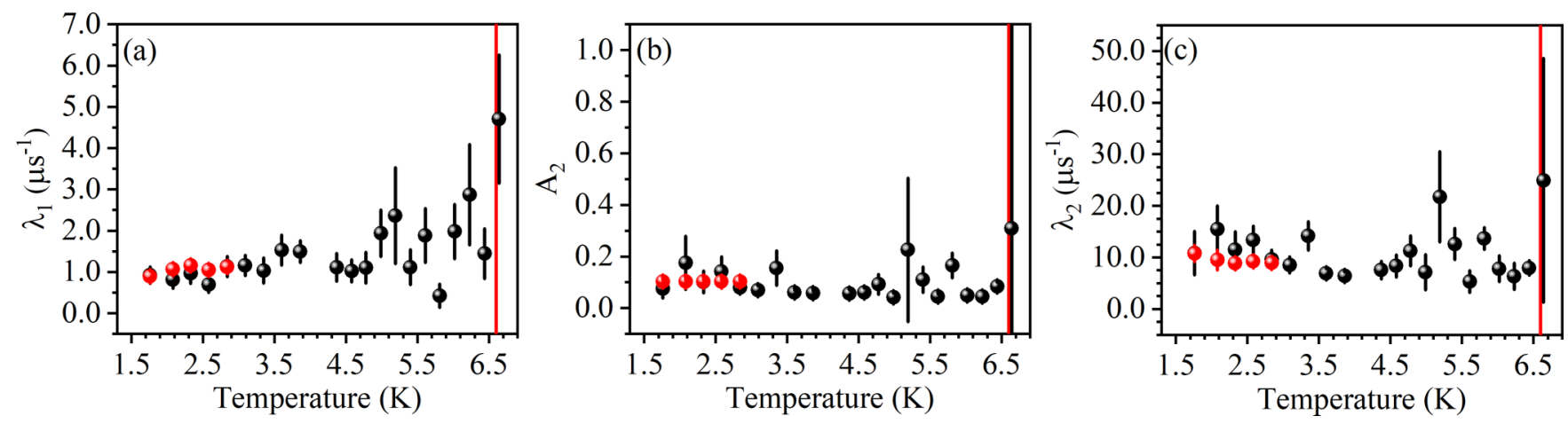

FIG. 11. Temperature dependencies of (a) $\lambda_{1}$, (b) $A_{2}$ and (c) $\lambda_{2}$ in Eq. (2). The red solid circles are fits based on the additional constraint presented in Appendix B. The red vertical line marks the position of $T_{\mathrm{N} 1}=6.6 \mathrm{~K}$, at which the $k_{1}$ spins undergo a phase transition and the fitting parameters become ill-defined. A similar effect has been reported in Ref. [19]. 
[1] F. D. M. Haldane, Nobel lecture: Topological quantum matter, Rev. Mod. Phys. 89, 040502 (2017).

[2] T. Giamarchi, Quantum Physics in One Dimension (Oxford University Press, Oxford, 2004).

[3] R. Coldea, D. A. Tennant, E. M. Wheeler, E. Wawrzynska, D. Prabhakaran, M. Telling, K. Habicht, P. Smeibidl, and K. Kiefer, Quantum criticality in an Ising chain: Experimental evidence for emergent E8 symmetry, Science 327, 177 (2010).

[4] B. Grenier, S. Petit, V. Simonet, E. Canévet, L.-P. Regnault, S. Raymond, B. Canals, C. Berthier, and P. Lejay, Longitudinal and Transverse Zeeman Ladders in the Ising-Like Chain Antiferromagnet $\mathrm{BaCo}_{2} \mathrm{~V}_{2} \mathrm{O}_{8}$, Phys. Rev. Lett. 114, 017201 (2015).

[5] M. Dupont, S. Capponi, N. Laflorencie, and E. Orignac, Dynamical response and dimensional crossover for spatially anisotropic antiferromagnets, Phys. Rev. B 98, 094403 (2018).

[6] K. Wierschem and P. Sengupta, Quenching the Haldane Gap in Spin-1 Heisenberg Antiferromagnets, Phys. Rev. Lett. 112, 247203 (2014).

[7] E. Dagotto, T. Hotta, and A. Moreo, Colossal magnetoresistant materials: the key role of phase separation, Phys. Rep. 344, 1 (2001).

[8] V. Emery and S. Kivelson, Frustrated electronic phase separation and high-temperature superconductors, Physica C 209, 597 (1993).

[9] S. Agrestini, C. L. Fleck, L. C. Chapon, C. Mazzoli, A. Bombardi, M. R. Lees, and O. A. Petrenko, Slow Magnetic Order-Order Transition in the Spin Chain Antiferromagnet $\mathrm{Ca}_{3} \mathrm{Co}_{2} \mathrm{O}_{6}$, Phys. Rev. Lett. 106, 197204 (2011).

[10] Y. Kamiya and C. D. Batista, Formation of Magnetic Microphases in $\mathrm{Ca}_{3} \mathrm{Co}_{2} \mathrm{O}_{6}$, Phys. Rev. Lett. 109, 067204 (2012).

[11] J. Schulenburg, A. Honecker, J. Schnack, J. Richter, and H.-J. Schmidt, Macroscopic Magnetization Jumps Due to Independent Magnons in Frustrated Quantum Spin Lattices, Phys. Rev. Lett. 88, 167207 (2002).

[12] M. S. S. Pereira, F. A. B. F. de Moura, and M. L. Lyra, Magnetocaloric effect in kinetically frustrated diamond chains, Phys. Rev. B 79, 054427 (2009).

[13] L. Shen, E. Jellyman, E. M. Forgan, E. Blackburn, M. Laver, E. Canévet, J. Schefer, Z. He, and M. Itoh, Unconventional magnetic phase separation in $\gamma-\mathrm{CoV}_{2} \mathrm{O}_{6}$, Phys. Rev. B 96, 054420 (2017).

[14] H. Mueller-Buschbaum and M. Kobel, On the crystal chemistry of the oxyvanadates $\mathrm{CoV}_{2} \mathrm{O}_{6}$ und $\mathrm{MnV}_{2} \mathrm{O}_{6}$, J. Alloys Compd. 176, 39 (1991).

[15] S. A. J. Kimber, H. Mutka, T. Chatterji, T. Hofmann, P. F. Henry, H. N. Bordallo, D. N. Argyriou, and J. P. Attfield, Metamagnetism and soliton excitations in the modulated ferromagnetic Ising chain $\mathrm{CoV}_{2} \mathrm{O}_{6}$, Phys. Rev. B 84, 104425 (2011).

[16] There are mixed reports on the magnetic modulation vectors in this system. In addition to what we have reported in Ref. [13], other wave vectors such as $k=\left(\frac{1}{4}, \frac{1}{2}, 0\right)$ [Lenertz et al., J. Phys. Chem. C 118, 13981 (2014)] and $k=\left(-\frac{1}{3}, 0, \frac{1}{3}\right)$ [Drees et al., Cryst. Growth Des. 15, 1168 (2015)] have also been reported, but we did not observe these additional modulations in our neutron diffraction study on a single crystal [13].

[17] P. D. de Réotier and A. Yaouanc, Muon spin rotation and relaxation in magnetic materials, J. Phys.: Condens. Matter 9, 9113 (1997).
[18] S. J. Blundell, Spin-polarized muons in condensed matter physics, Contemp. Phys. 40, 175 (1999).

[19] M. L. Brooks, T. Lancaster, S. J. Blundell, W. Hayes, F. L. Pratt, and Z. Fisk, Magnetic phase separation in $\mathrm{EuB}_{6}$ detected by muon spin rotation, Phys. Rev. B 70, 020401(R) (2004).

[20] P. M. S. Monteiro, P. J. Baker, A. Ionescu, C. H. W. Barnes, Z. Salman, A. Suter, T. Prokscha, and S. Langridge, Spatially Homogeneous Ferromagnetism Below the Enhanced Curie Temperature in $\mathrm{EuO}_{1-x}$ Thin Films, Phys. Rev. Lett. 110, 217208 (2013).

[21] V. G. Storchak, D. G. Eshchenko, E. Morenzoni, T. Prokscha, A. Suter, X. Liu, and J. K. Furdyna, Spatially Resolved Inhomogeneous Ferromagnetism in (Ga, Mn)As Diluted Magnetic Semiconductors: A Microscopic Study by Muon Spin Relaxation, Phys. Rev. Lett. 101, 027202 (2008).

[22] Z. He, J.-I. Yamaura, Y. Ueda, and W. Cheng, Magnetic properties of $\mathrm{Co}_{2} \mathrm{~V}_{2} \mathrm{O}_{7}$ single crystals grown by flux method, J. Solid State Chem. 182, 2526 (2009).

[23] M. Månsson, K. Prša, J. Sugiyama, H. Nozaki, A. Amato, K. Omura, S. Kimura, and M. Hagiwara, Microscopic magnetic nature of the quasi-one-dimensional antiferromagnet $\mathrm{BaCo}_{2} \mathrm{~V}_{2} \mathrm{O}_{8}$, Phys. Procedia 30, 146 (2012).

[24] T. Lancaster, S. J. Blundell, P. J. Baker, M. L. Brooks, W. Hayes, F. L. Pratt, R. Coldea, T. Sörgel, and M. Jansen, Anomalous Temperature Evolution of the Internal Magnetic Field Distribution in the Charge-Ordered Triangular Antiferromagnet $\mathrm{AgNiO}_{2}$, Phys. Rev. Lett. 100, 017206 (2008).

[25] J. Sugiyama, H. Nozaki, M. Harada, K. Kamazawa, O. Ofer, M. Månsson, J. H. Brewer, E. J. Ansaldo, K. H. Chow, Y. Ikedo, Y. Miyake, K. Ohishi, I. Watanabe, G. Kobayashi, and R. Kanno, Magnetic and diffusive nature of $\mathrm{LiFePO}_{4}$ investigated by muon spin rotation and relaxation, Phys. Rev. B 84, 054430 (2011).

[26] Fits including the initial fast relaxation give similar values for the parameters used in Eq. (3) but with a worse quality of fit. Since the wTF measurements are used to determine the nature of the $A_{\mathrm{C}}^{\mathrm{ZF}}$ term in Eqs. (1) and (2), the simplified model in Eq. (3) is sufficient for this purpose.

[27] F. L. Pratt, Field dependence of $\mu$ SR signals in a polycrystalline magnet, J. Phys.: Condens. Matter 19, 456207 (2007).

[28] L. Clark, G. Sala, D. D. Maharaj, M. B. Stone, K. S. Knight, M. T. F. Telling, X. Wang, X. Xu, J. Kim, Y. Li, S.-W. Cheong, and B. D. Gaulin, Two-dimensional spin liquid behavior in the triangular-honeycomb antiferromagnet $\mathrm{TbInO}_{3}$, Nat. Phys. 15, 262 (2019).

[29] P. J. Baker, J. S. Möller, F. L. Pratt, W. Hayes, S. J. Blundell, T. Lancaster, T. F. Qi, and G. Cao, Weak magnetic transitions in pyrochlore $\mathrm{Bi}_{2} \mathrm{Ir}_{2} \mathrm{O}_{7}$, Phys. Rev. B 87, 180409(R) (2013).

[30] A. K. Bera, B. Lake, F. H. L. Essler, L. Vanderstraeten, C. Hubig, U. Schollwöck, A. T. M. N. Islam, A. Schneidewind, and D. L. Quintero-Castro, Spinon confinement in a quasi-onedimensional anisotropic Heisenberg magnet, Phys. Rev. B 96, 054423 (2017).

[31] G. Lawes, M. Kenzelmann, N. Rogado, K. H. Kim, G. A. Jorge, R. J. Cava, A. Aharony, O. Entin-Wohlman, A. B. Harris, T. Yildirim, Q. Z. Huang, S. Park, C. Broholm, and A. P. Ramirez, Competing Magnetic Phases on a Kagomé Staircase, Phys. Rev. Lett. 93, 247201 (2004).

[32] https://doi.org/10.5286/ISIS.E.RB1910224. 
[33] J. Rodríguez-Carvajal, Recent advances in magnetic structure determination by neutron powder diffraction, Physica B 192, 55 (1993).

[34] F. L. Pratt, Repolarization of anisotropic muonium in orientationally disordered solids, Philos. Mag. Lett. 75, 371 (1997).
[35] Y. Ikedo, J. Sugiyama, H. Nozaki, H. Itahara, J. H. Brewer, E. J. Ansaldo, G. D. Morris, D. Andreica, and A. Amato, Spatial inhomogeneity of magnetic moments in the cobalt oxide spinel $\mathrm{Co}_{3} \mathrm{O}_{4}$, Phys. Rev. B 75, 054424 (2007). 